\title{
3 Research Square \\ Renal Function during Hospitalization and Outcome in Chinese Patients with Acute Decompensated Heart Failure
}

Hao-Wei Lee

Taipei Veterans General Hospital

Chin-Chou Huang ( $\nabla$ cchuang4@vghtpe.gov.tw)

Taipei Veterans General Hospital

Chih-Yu Yang

National Yang Ming Chiao Tung University

Hsin-Bang Leu

Taipei Veterans General Hospital

Po-Hsun Huang

Taipei Veterans General Hospital

Tao-Cheng Wu

Taipei Veterans General Hospital

Shing-Jong Lin

Taipei Veterans General Hospital

Jaw-Wen Chen

Taipei Veterans General Hospital

\section{Research Article}

Keywords: chronic kidney disease, heart failure, hospitalization, outcome, renal function, worsening renal function

Posted Date: December 6th, 2021

DOI: https://doi.org/10.21203/rs.3.rs-1098724/v1

License: (c) (i) This work is licensed under a Creative Commons Attribution 4.0 International License.

Read Full License 


\section{Abstract}

It is well known that the heart and kidney have a bi-directional correlation, in which organ dysfunction results in maladaptive changes in the other. We aimed to investigate the impact of renal function and its decline during hospitalization on clinical outcomes in patients with acute decompensated heart failure (ADHF). A total of 119 consecutive Chinese patients admitted for ADHF were prospectively enrolled. The course of renal function was presented with estimated glomerular filtration rate (eGFR), calculated by the four-variable equation proposed by the Modification of Diet in Renal Disease (MDRD) Study. Worsening

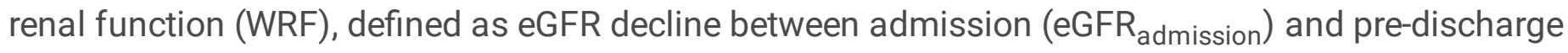
(eGFR predischarge $)$, occurred in 41 patients. Clinical outcomes during the follow-up period were defined as 4P-major adverse cardiovascular events (4P-MACE), including the composition of cardiovascular death, nonfatal myocardial infarction, nonfatal stroke, and nonfatal HF hospitalization. During an average follow-up period of 2.6 \pm 3.2 years, 66 patients experienced 4P-MACE. Cox regression analysis revealed

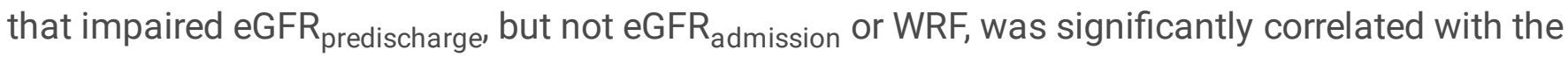
development of 4P-MACE (HR, 2.003; 95\% Cl, 1.072-3.744; $P=0.029)$. In conclusion, impaired renal function before discharge, but not WRF, is a significant risk factor for poor outcomes in patients with ADHF.

\section{Introduction}

It is well known that the heart and kidney have a bi-directional correlation, in which one organ dysfunction results in maladaptive change in the other. ${ }^{1}$ The prevalence of renal insufficiency ranges from $20-57 \%$ in patients with chronic stable heart failure (HF) and acute decompensated heart failure (ADHF). ${ }^{2}$ Compared to patients without chronic kidney disease (CKD), patients with both HF and CKD may have up to a $50 \%$ greater risk of mortality, especially patients of HF with reduced ejection fraction (HFrEF). ${ }^{3}$ In addition to mortality, patients with CKD hospitalized with $\mathrm{HF}$ also have a greater risk of CKD progression. ${ }^{4}$

The impact of WRF on the clinical outcomes in patients with ADHF remains controversial. Some studies have shown that worsening renal function (WRF) in patients admitted due to ADHF was associated with higher in-hospital mortality, longer hospital stay, readmission, higher mortality after discharge, and higher costs. ${ }^{5-13}$ However, some studies have shown that patients with WRF have similar mortality and rehospitalization rates to those without WRF. ${ }^{14-18}$ Furthermore, there is scarce information regarding the impact of WRF in Chinese patients. Therefore, we aimed to investigate the impact of renal function and its changes during hospitalization on clinical outcomes in Chinese patients admitted for ADHF.

\section{Methods}

\section{Participants}


Consecutive Chinese patients admitted for ADHF were enrolled at the Taipei Veterans General Hospital between June 1, 2008, and April 31, 2010. ADHF was defined according to current guidelines. ${ }^{19,20}$ Patients with severe comorbidity, end-stage renal disease (ESRD), moderate or severe pulmonary disease, malignancy, uncontrolled thyroid disease, drug or alcohol abuse, infections, or an inflammatory illness such as sepsis, arthritis, or connective tissue disease were excluded. Patients with acute coronary syndrome, significant aortic valve disease, myocarditis, infiltrative or hypertrophic cardiomyopathy, uncontrolled tachyarrhythmias, in need of a mechanical assist device, or having significant congenital heart disease were also excluded. The study protocol was approved by the Ethics Committee of Taipei Veterans General Hospital. All patients agreed to participate and signed the informed consent document for the study. This study was conducted in accordance with the principles of the Declaration of Helsinki.

\section{Study design}

The study included a comprehensive examination of each participant's patient history and physical examination. Patient comorbidities were recorded, including hypertension (HTN), diabetes mellitus (DM), atrial fibrillation (AF), and hyperlipidemia. Patients were defined as having ischemic heart disease (IHD) if their coronary angiography showed a $\geq 70 \%$ luminal diameter and narrowing in at least one major epicardial coronary artery, or if there was documented myocardial infarction or HF secondary to postinfarction ventricular aneurysm. Echocardiography was performed, and left ventricular ejection fraction (LVEF) was measured. Patients with LVEF $<40 \%$ were defined as having HFrEF.

Pre-discharge medication prescriptions for HF were recorded, and these included diuretics, angiotensinconverting enzyme inhibitors (ACEI)/angiotensin receptor blockers (ARB), beta-blockers, digitalis, and vasodilators.

\section{Laboratory measurements}

Fasting whole blood samples of the patients were obtained by venipuncture. The blood samples were centrifuged, and the serum was used for analysis. Cardiac troponin-I levels were determined using an Abbott Axsym system (Abbott Laboratories, Abbott Park, IL, USA). The N-terminal pro-brain natriuretic peptide (NT-pro-BNP) was measured using the Roche Elecsys NT-proBNP (Roche Diagnostics GmbH, Indianapolis, IN, USA). High-sensitivity C-reactive protein (hs-CRP) levels were determined with a validated, high-sensitivity assay using an autoanalyzer (IMMAGE Immunochemistry Systems, Beckman Coulter, Inc., Brea, CA, USA).

A series of studies on serum creatinine levels during hospitalization were performed. Estimated glomerular filtration rate (eGFR) was calculated using the four-variable equation proposed by the Modification of Diet in Renal Disease (MDRD) Study. ${ }^{21}$ Patients were further divided into different groups

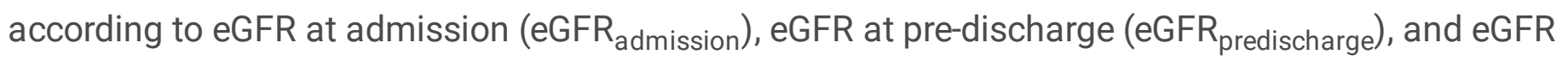
change between admission and pre-discharge. According to eGFR $\mathrm{R}_{\text {admission }}$ levels, patients were divided

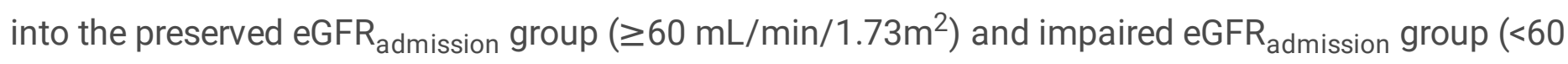
$\mathrm{mL} / \mathrm{min} / 1.73 \mathrm{~m}^{2}$ ). According to eGFR predischarge levels, patients were divided into the preserved 


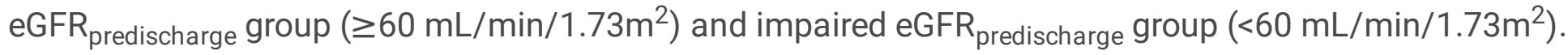
WRF was defined as an eGFR decline between admission and pre-discharge.

\section{Clinical outcomes}

Clinical outcomes during the follow-up period were defined as 4P-major adverse cardiovascular events (4P-MACE), including the composition of cardiovascular (CV) death, nonfatal myocardial infarction (MI), nonfatal stroke, and nonfatal HF hospitalization.

\section{Statistical Analysis}

Statistical analysis was performed using the Statistical Package for Social Sciences software (version 21.0, SPSS Inc., Chicago, IL, USA). All data are expressed as the mean \pm standard deviation or frequency (percentage). Parametric continuous data between different patient groups were compared using the unpaired Student's t-test, and nonparametric data were compared using the Mann-Whitney test. Categorical variables were analyzed using the Chi-squared test or Fisher's exact test. Survival analysis was assessed using the Kaplan-Meier curve, with significance based on the log-rank test. To assess the independent effects of renal function (impaired eGFR ${ }_{\text {admission, }}$ impaired eGFR predischarge, and eGFR decline) and 4P-MACE, Cox proportional hazard regression analysis was performed. The adjusted hazard ratios (HRs) with 95\% confidence intervals (Cls) were estimated after adjusting for potential confounding factors. The HRs of renal function for 4P-MACE were adjusted for age, sex, HTN, DM, ischemic heart disease (IHD), and use of antihypertensive drugs. Statistical significance was defined as a two-sided $P$ value $<0.05$.

\section{Results}

\section{Baseline characteristics}

A total of 119 patients with ADHF were eligible for enrollment. The mean age of the participants was $73.5 \pm 12.9$ years, and approximately $76.5 \%$ were men. The average body mass index was $23.9 \pm 4.5 \mathrm{~kg} / \mathrm{m}^{2}$. The comorbidities included HTN in 92 patients (77.3\%), DM in 48 patients (40.3\%), AF in 57 patients (47.9\%), and hyperlipidemia in 29 patients (24.4\%). Among these patients, $60(50.4 \%)$ had ischemic heart disease. There were 58 patients (48.7\%) with HFrEF, and the mean LVEF was $40.3 \pm 15.5 \%$. Pre-discharge medications included diuretics (79.8\%), ACEI/ARB (71.4\%), beta-blockers (47.1\%), digitalis $(22.7 \%)$, and vasodilators $(52.9 \%)$. The renal function of the participants upon admission was a serum creatinine level of $1.7 \pm 0.9 \mathrm{mg} / \mathrm{dL}$ and an eGFR admission of $50.7 \pm 25.9 \mathrm{~mL} / \mathrm{min} / 1.73 \mathrm{~m}^{2}$. The renal function of the participants before discharge was a serum creatinine level of $1.5 \pm 0.7 \mathrm{mg} / \mathrm{dL}$ and an eGFR predischarge $_{\text {of }}$ $59.2 \pm 31.3 \mathrm{~mL} / \mathrm{min} / 1.73 \mathrm{~m}^{2}$. The average change in eGFR was $0.2 \pm 0.5 \mathrm{~mL} / \mathrm{min} / 1.73 \mathrm{~m}^{2}$ (Table 1 ). 
Table 1

Baseline characteristics of the patients

All $(n=119)$

Age, years

Male, $\mathrm{n}(\%)$

$\mathrm{BMl}, \mathrm{kg} / \mathrm{m}^{2}$

Smoking, $\mathrm{n}(\%)$

HTN, n(\%)

$\mathrm{DM}, \mathrm{n}(\%)$

$A F, n(\%)$

Hyperlipidemia, $\mathrm{n}(\%)$

IHD, $\mathrm{n}(\%)$

HFrEF, $n(\%)$

LVEF, \%

Laboratory data

HDL-C, mg/dL

LDL-C, mg/dL

hs-CRP, mg/dL

Troponin-l, ng/mL

NT-pro-BNP, pg/ml

Creatinine $_{\text {admission }}, \mathrm{mg} / \mathrm{dL}$

Creatinine $_{\text {predischarge, }} \mathrm{mg} / \mathrm{dL}$

eGFR admission $_{\text {}} \mathrm{mL} / \mathrm{min} / 1.73 \mathrm{~m}^{2}$

eGFR predischarge, $\mathrm{mL} / \mathrm{min} / 1.73 \mathrm{~m}^{2}$

eGFR change, $\mathrm{mL} / \mathrm{min} / 1.73 \mathrm{~m}^{2}$
$73.5 \pm 12.9$

$91(76.5 \%)$

$23.9 \pm 4.5$

27 (22.7\%)

92 (77.3\%)

48 (40.3\%)

57 (47.9\%)

$29(24.4 \%)$

60 (50.4\%)

$58(48.7 \%)$

$40.3 \pm 15.5$
$43.2 \pm 19.0$

$88.4 \pm 30.3$

$1.2 \pm 1.4$

$0.1 \pm 0.2$

$4006.2 \pm 4036.4$

$1.7 \pm 0.9$

$1.5 \pm 0.7$

$50.7 \pm 25.9$

$59.2 \pm 31.3$

$0.2 \pm 0.5$

ACEl, angiotensin-converting enzyme inhibitor; $A F$, atrial fibrillation; $A R B$, angiotensin receptor blocker; $\mathrm{BMI}$, body mass index; DM, diabetes mellitus; eGFR, estimated glomerular filtration rate; HDL-C, highdensity lipoprotein-cholesterol; HFrEF, heart failure with reduced ejection fraction; $\mathrm{HTN}$, hypertension; hs-CRP, high sensitivity C-reactive protein; IHD, ischemic heart disease; LDL-C, low-density lipoproteincholesterol; LVEF, left ventricular ejection fraction; NT-pro-BNP, N-terminal pro-brain natriuretic peptide. 
All $(n=119)$

Predischarge medication

Diuretics, n(\%)

$95(79.8 \%)$

ACEI/ARB, $n(\%)$

$85(71.4 \%)$

Beta-blockers, $\mathrm{n}(\%)$

$56(47.1 \%)$

Digitalis, $\mathrm{n}(\%)$

$27(22.7 \%)$

Vasodilators, $\mathrm{n}(\%)$

$63(52.9 \%)$

Follow-up duration (years)

$2.6 \pm 3.2$

ACEl, angiotensin-converting enzyme inhibitor; $A F$, atrial fibrillation; $A R B$, angiotensin receptor blocker; BMI, body mass index; DM, diabetes mellitus; eGFR, estimated glomerular filtration rate; HDL-C, highdensity lipoprotein-cholesterol; HFrEF, heart failure with reduced ejection fraction; $\mathrm{HTN}$, hypertension; hs-CRP, high sensitivity C-reactive protein; IHD, ischemic heart disease; LDL-C, low-density lipoproteincholesterol; LVEF, left ventricular ejection fraction; NT-pro-BNP, N-terminal pro-brain natriuretic peptide.

Based on renal function upon admission, there were 35 patients with preserved GFR $_{\text {admission }}(\geq 60$ $\left.\mathrm{mL} / \mathrm{min} / 1.73 \mathrm{~m}^{2}\right)$ and 84 patients with impaired eGFR admission $\left(<60 \mathrm{~mL} / \mathrm{min} / 1.73 \mathrm{~m}^{2}\right)$. When comparing to those with preserved eGFR admission, patients with impaired eGFR admission had more HTN (83.3\% vs. 62.9\%, $P=0.015)$, DM (47.6\% vs. $22.9 \%, P=0.012)$, and ischemic heart disease ( $57.1 \%$ vs. $34.3 \%, P=0.023$ ), and used more vasodilators than patients with preserved eGFR admission $(59.5 \%$ vs. $37.1 \%, P=0.026)$. The mean eGFR $\mathrm{admission}_{\text {was }} 37.9 \pm 13.3 \mathrm{~mL} / \mathrm{min} / 1.73 \mathrm{~m}^{2}$ in patients with impaired $\mathrm{eGFR}_{\text {admission }}$ and $81.3 \pm 23.1$ $\mathrm{mL} / \mathrm{min} / 1.73 \mathrm{~m}^{2}$ in patients with preserved eGFR ${ }_{\text {admission }}(P<0.001)$. The mean eGFR predischarge was $46.6 \pm 20.4 \mathrm{~mL} / \mathrm{min} / 1.73 \mathrm{~m}^{2}$ in patients with impaired eGFR $\mathrm{admission}$ and $89.5 \pm 32.5 \mathrm{~mL} / \mathrm{min} / 1.73 \mathrm{~m}^{2}$ in patients with preserved eGFR admission $(P<0.001)$. The changes in eGFR between admission and discharge were similar in the two groups (Table 2). 
Table 2

Baseline characteristics according to admission renal function

\begin{tabular}{|c|c|c|c|}
\hline & $\begin{array}{l}\text { Preserved eGFR } \text { admission } \\
(n=35)\end{array}$ & $\begin{array}{l}\text { Impaired eGFR }{ }_{\text {admission }} \\
(n=84)\end{array}$ & $P$-value \\
\hline Age, years & $71.8 \pm 13.7$ & $74.3 \pm 12.5$ & 0.357 \\
\hline Male, $\mathrm{n}(\%)$ & $26(74.3 \%)$ & $65(77.4 \%)$ & 0.717 \\
\hline $\mathrm{BMI}, \mathrm{kg} / \mathrm{m}^{2}$ & $22.6 \pm 2.7$ & $24.4 \pm 4.9$ & 0.131 \\
\hline Smoking, $\mathrm{n}(\%)$ & $8(22.9 \%)$ & $19(22.6 \%)$ & 0.977 \\
\hline HTN, n(\%) & $22(62.9 \%)$ & $70(83.3 \%)$ & 0.015 \\
\hline $\mathrm{DM}, \mathrm{n}(\%)$ & $8(22.9 \%)$ & $40(47.6 \%)$ & 0.012 \\
\hline$A F, n(\%)$ & $20(57.1 \%)$ & $37(44.0 \%)$ & 0.193 \\
\hline Hyperlipidemia, n(\%) & $5(14.3 \%)$ & $24(28.6 \%)$ & 0.098 \\
\hline $\mathrm{IHD}, \mathrm{n}(\%)$ & $12(34.3 \%)$ & $48(57.1 \%)$ & 0.023 \\
\hline HFrEF, n(\%) & $21(60.0 \%)$ & $37(44.0 \%)$ & 0.113 \\
\hline LVEF, \% & $36.5 \pm 15.7$ & $41.7 \pm 15.3$ & 0.111 \\
\hline \multicolumn{4}{|l|}{ Laboratory data } \\
\hline $\mathrm{HDL}-\mathrm{C}, \mathrm{mg} / \mathrm{dL}$ & $47.4 \pm 21.0$ & $41.3 \pm 18.0$ & 0.212 \\
\hline LDL-C, mg/dL & $84.4 \pm 28.5$ & $90.2 \pm 31.1$ & 0.398 \\
\hline hs-CRP, mg/dL & $1.1 \pm 1.2$ & $1.2 \pm 1.5$ & 0.732 \\
\hline Troponin-I, ng/mL & $0.2 \pm 0.2$ & $0.1 \pm 0.2$ & 0.385 \\
\hline NT-pro-BNP, pg/ml & $4112.1 \pm 5965.6$ & $3971.5 \pm 3232.3$ & 0.921 \\
\hline Creatinine $_{\text {admission }}, \mathrm{mg} / \mathrm{dL}$ & $1.0 \pm 0.2$ & $2.0 \pm 0.8$ & $<0.001$ \\
\hline Creatinine $_{\text {predischarge, }}, \mathrm{mg} / \mathrm{dL}$ & $0.9 \pm 0.3$ & $1.7 \pm 0.7$ & $<0.001$ \\
\hline $\mathrm{eGFR}_{\mathrm{admission}}, \mathrm{mL} / \mathrm{min} / 1.73 \mathrm{~m}^{2}$ & $81.3 \pm 23.1$ & $37.9 \pm 13.3$ & $<0.001$ \\
\hline eGFR predischarge, $\mathrm{mL} / \mathrm{min} / 1.73 \mathrm{~m}^{2}$ & $89.5 \pm 32.5$ & $46.6 \pm 20.4$ & $<0.001$ \\
\hline
\end{tabular}

ACEl, angiotensin-converting enzyme inhibitor; $A F$, atrial fibrillation; $A R B$, angiotensin receptor blocker; $\mathrm{BMI}$, body mass index; DM, diabetes mellitus; eGFR, estimated glomerular filtration rate; HDL-C, highdensity lipoprotein-cholesterol; HFrEF, heart failure with reduced ejection fraction; $\mathrm{HTN}$, hypertension; hs-CRP, high sensitivity C-reactive protein; IHD, ischemic heart disease; LDL-C, low-density lipoproteincholesterol; LVEF, left ventricular ejection fraction; NT-pro-BNP, N-terminal pro-brain natriuretic peptide. 


\begin{tabular}{|c|c|c|c|}
\hline & $\begin{array}{l}\text { Preserved eGFR } \text { admission } \\
(\mathrm{n}=35)\end{array}$ & $\begin{array}{l}\text { Impaired eGFR admission } \\
(\mathrm{n}=84)\end{array}$ & $P$-value \\
\hline eGFR change, $\mathrm{mL} / \mathrm{min} / 1.73 \mathrm{~m}^{2}$ & $0.1 \pm 0.3$ & $0.3 \pm 0.6$ & 0.065 \\
\hline \multicolumn{4}{|l|}{ Predischarge medication } \\
\hline Diuretics, n(\%) & $29(82.9 \%)$ & $66(78.6 \%)$ & 0.595 \\
\hline ACEI/ARB, n(\%) & $29(82.9 \%)$ & $56(66.7 \%)$ & 0.075 \\
\hline Beta-blockers, n(\%) & $17(48.6 \%)$ & $39(46.4 \%)$ & 0.831 \\
\hline Digitalis, $\mathrm{n}(\%)$ & $12(34.3 \%)$ & $15(17.9 \%)$ & 0.051 \\
\hline Vasodilators, n(\%) & $13(37.1 \%)$ & $50(59.5 \%)$ & 0.026 \\
\hline Follow-up duration (years) & $3.3 \pm 3.7$ & $2.3 \pm 2.9$ & 0.126 \\
\hline \multicolumn{4}{|c|}{$\begin{array}{l}\text { ACEl, angiotensin-converting enzyme inhibitor; AF, atrial fibrillation; ARB, angiotensin receptor blocker; } \\
\text { BMI, body mass index; DM, diabetes mellitus; eGFR, estimated glomerular filtration rate; HDL-C, high- } \\
\text { density lipoprotein-cholesterol; HFrEF, heart failure with reduced ejection fraction; HTN, hypertension; } \\
\text { hs-CRP, high sensitivity C-reactive protein; IHD, ischemic heart disease; LDL-C, low-density lipoprotein- } \\
\text { cholesterol; LVEF, left ventricular ejection fraction; NT-pro-BNP, N-terminal pro-brain natriuretic peptide. }\end{array}$} \\
\hline
\end{tabular}

According to the renal function before discharge, 51 patients had preserved eGFR predischarge $(\geq 60$ $\left.\mathrm{mL} / \mathrm{min} / 1.73 \mathrm{~m}^{2}\right)$ and 68 patients with impaired eGFR predischarge $\left(<60 \mathrm{~mL} / \mathrm{min} / 1.73 \mathrm{~m}^{2}\right)$. Compared to patients with preserved eGFR predischarge, $_{\text {, patients with impaired eGFR }}$ predischarge had more DM (51.5\% vs. $25.5 \%, P=0.004)$ and ischemic heart disease (58.8\% vs. $39.2 \%, P=0.034)$, and used more diuretics ( $86.8 \%$ vs. $70.6 \%, P=0.030)$ and less digitalis $(11.8 \%$ vs. $37.3 \%, P=0.001)$ than patients with preserved eGFR predischarge. The mean eGFR admission $_{\text {was }} 37.2 \pm 14.5 \mathrm{~mL} / \mathrm{min} / 1.73 \mathrm{~m}^{2}$ in patients with impaired $\mathrm{eGFR}_{\text {predischarge }}$ and $68.6 \pm 27.0 \mathrm{~mL} / \mathrm{min} / 1.73 \mathrm{~m}^{2}$ in patients with preserved eGFR predischarge $(P<0.001)$. The mean eGFR predischarge was $39.0 \pm 12.5 \mathrm{~mL} / \mathrm{min} / 1.73 \mathrm{~m}^{2}$ in patients with impaired eGFR $\mathrm{predischarge}$ and $86.2 \pm 28.5 \mathrm{~mL} / \mathrm{min} / 1.73 \mathrm{~m}^{2}$ in patients with preserved eGFR predischarge $(P<0.001)$. Patients with preserved

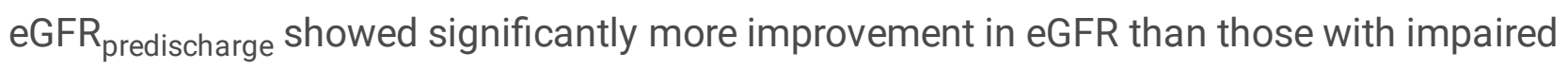
eGFR $_{\text {predischarge }}\left(0.4 \pm 0.6 \mathrm{~mL} / \mathrm{min} / 1.73 \mathrm{~m}^{2}\right.$ vs. $\left.0.1 \pm 0.4 \mathrm{~mL} / \mathrm{min} / 1.73 \mathrm{~m}^{2}, P=0.012\right)$ (Table 3). 
Table 3

Baseline characteristics according to pre-discharge renal function

\begin{tabular}{|c|c|c|c|}
\hline & $\begin{array}{l}\text { Preserved eGFR } \text { predischarge } \\
(n=51)\end{array}$ & $\begin{array}{l}\text { Impaired eGFR } \text { predischarge } \\
(n=68)\end{array}$ & $P$-value \\
\hline Age, years & $71.2 \pm 15.7$ & $75.3 \pm 10.1$ & 0.086 \\
\hline Male, n(\%) & 39 (76.5\%) & $52(76.5 \%)$ & $>0.999$ \\
\hline $\mathrm{BMI}, \mathrm{kg} / \mathrm{m}^{2}$ & $23.9 \pm 4.1$ & $24.0 \pm 4.8$ & 0.907 \\
\hline Smoking, $\mathrm{n}(\%)$ & $13(25.5 \%)$ & $14(20.6 \%)$ & 0.527 \\
\hline HTN, n(\%) & $38(74.5 \%)$ & $54(79.4 \%)$ & 0.527 \\
\hline $\mathrm{DM}, \mathrm{n}(\%)$ & $13(25.5 \%)$ & $35(51.5 \%)$ & 0.004 \\
\hline$A F, n(\%)$ & $27(52.9 \%)$ & $30(44.1 \%)$ & 0.340 \\
\hline Hyperlipidemia, n(\%) & $9(17.6 \%)$ & $20(29.4 \%)$ & 0.139 \\
\hline $\mathrm{IHD}, \mathrm{n}(\%)$ & $20(39.2 \%)$ & $40(58.8 \%)$ & 0.034 \\
\hline HFrEF, n(\%) & $30(58.8 \%)$ & $29(41.2 \%)$ & 0.057 \\
\hline LVEF, \% & $38.8 \pm 14.7$ & $41.4 \pm 16.1$ & 0.543 \\
\hline \multicolumn{4}{|l|}{ Laboratory data } \\
\hline $\mathrm{HDL}-\mathrm{C}, \mathrm{mg} / \mathrm{dL}$ & $46.6 \pm 21.1$ & $40.3 \pm 16.8$ & 0.144 \\
\hline LDL-C, mg/dL & $84.4 \pm 28.3$ & $91.7 \pm 31.8$ & 0.261 \\
\hline hs-CRP, mg/dL & $1.1 \pm 1.2$ & $1.2 \pm 1.6$ & 0.711 \\
\hline Troponin-I, ng/mL & $0.1 \pm 0.2$ & $0.1 \pm 0.2$ & 0.940 \\
\hline NT-pro-BNP, pg/ml & $3838.6 \pm 4905.4$ & $4115.7 \pm 3403.2$ & 0.782 \\
\hline Creatinine $_{\text {admission }}, \mathrm{mg} / \mathrm{dL}$ & $1.2 \pm 0.5$ & $2.1 \pm 0.9$ & $<0.001$ \\
\hline Creatinine $_{\text {predischarge, }}, \mathrm{mg} / \mathrm{dL}$ & $0.9 \pm 0.2$ & $1.9 \pm 0.7$ & $<0.001$ \\
\hline $\mathrm{eGFR}_{\text {admission }}, \mathrm{mL} / \mathrm{min} / 1.73 \mathrm{~m}^{2}$ & $68.6 \pm 27.0$ & $37.2 \pm 14.5$ & $<0.001$ \\
\hline eGFR predischarge, $\mathrm{mL} / \mathrm{min} / 1.73 \mathrm{~m}^{2}$ & $86.2 \pm 28.5$ & $39.0 \pm 12.5$ & $<0.001$ \\
\hline
\end{tabular}

ACEl, angiotensin-converting enzyme inhibitor; $A F$, atrial fibrillation; $A R B$, angiotensin receptor blocker; $\mathrm{BMI}$, body mass index; DM, diabetes mellitus; eGFR, estimated glomerular filtration rate; HDL-C, highdensity lipoprotein-cholesterol; HFrEF, heart failure with reduced ejection fraction; $\mathrm{HTN}$, hypertension; hs-CRP, high sensitivity C-reactive protein; IHD, ischemic heart disease; LDL-C, low-density lipoproteincholesterol; LVEF, left ventricular ejection fraction; NT-pro-BNP, N-terminal pro-brain natriuretic peptide. 


\begin{tabular}{|c|c|c|c|}
\hline & $\begin{array}{l}\text { Preserved eGFR } R_{\text {predischarge }} \\
(n=51)\end{array}$ & $\begin{array}{l}\text { Impaired eGFR } \text { predischarge } \\
(n=68)\end{array}$ & $P$-value \\
\hline eGFR change, $\mathrm{mL} / \mathrm{min} / 1.73 \mathrm{~m}^{2}$ & $0.4 \pm 0.6$ & $0.1 \pm 0.4$ & 0.012 \\
\hline \multicolumn{4}{|l|}{ Predischarge medication } \\
\hline Diuretics, n(\%) & $36(70.6 \%)$ & $59(86.8 \%)$ & 0.030 \\
\hline ACEI/ARB, n(\%) & $39(76.5 \%)$ & $46(67.6 \%)$ & 0.292 \\
\hline Beta-blockers, n(\%) & $25(49.0 \%)$ & $31(45.6 \%)$ & 0.711 \\
\hline Digitalis, $\mathrm{n}(\%)$ & $19(37.3 \%)$ & $8(11.8 \%)$ & 0.001 \\
\hline Vasodilators, n(\%) & $22(43.1 \%)$ & $41(60.3 \%)$ & 0.064 \\
\hline Follow-up duration (years) & $3.6 \pm 3.7$ & $1.9 \pm 2.5$ & 0.004 \\
\hline \multicolumn{4}{|c|}{$\begin{array}{l}\text { ACEl, angiotensin-converting enzyme inhibitor; AF, atrial fibrillation; ARB, angiotensin receptor blocker; } \\
\text { BMI, body mass index; DM, diabetes mellitus; eGFR, estimated glomerular filtration rate; HDL-C, high- } \\
\text { density lipoprotein-cholesterol; HFrEF, heart failure with reduced ejection fraction; HTN, hypertension; } \\
\text { hs-CRP, high sensitivity C-reactive protein; IHD, ischemic heart disease; LDL-C, low-density lipoprotein- } \\
\text { cholesterol; LVEF, left ventricular ejection fraction; NT-pro-BNP, N-terminal pro-brain natriuretic peptide. }\end{array}$} \\
\hline
\end{tabular}

Other biomarkers, cardiac troponin-I, NT-pro-BNP, and hs-CRP, were similar in patients with preserved or impaired renal function according to either eGFR admission $_{\text {or }}$ GFR predischarge $_{\text {. }}$

\section{Renal function changes during hospitalization}

We further analyzed the changes in the eGFR between admission and discharge. There were 41 patients with eGFR decline and 78 without eGFR decline. When compared to those without eGFR decline, patients with eGFR decline used more diuretics $(90.2 \%$ vs. $74.4 \%, P=0.040)$ but less digitalis $(12.2 \%$ vs. $28.2 \%$, $P=0.048)$. The mean eGFR admission was similar in the two groups. However, patients with eGFR decline had lower eGFR predischarge $_{\text {than }}$ those without eGFR decline $\left(46.8 \pm 25.6 \mathrm{~mL} / \mathrm{min} / 1.73 \mathrm{~m}^{2} \mathrm{vs} .65 .8 \pm 32.2\right.$ $\left.\mathrm{mL} / \mathrm{min} / 1.73 \mathrm{~m}^{2}, P=0.001\right)$. The changes in eGFR in the two groups were $-0.2 \pm 0.1 \mathrm{~mL} / \mathrm{min} / 1.73 \mathrm{~m}^{2}$ and $0.4 \pm 0.5 \mathrm{~mL} / \mathrm{min} / 1.73 \mathrm{~m}^{2}(P<0.001)$, respectively (Table 4). Cardiac troponin-I, NT-pro-BNP, and hs-CRP levels were similar in patients with and without eGFR decline. 
Table 4

Baseline characteristics according to renal function changes during hospitalization

\begin{tabular}{|c|c|c|c|}
\hline & No eGFR decline $(n=78)$ & eGFR decline $(n=41)$ & $P$-value \\
\hline Age, years & $72.4 \pm 13.8$ & $75.8 \pm 10.8$ & 0.144 \\
\hline Male, n(\%) & $60(76.9 \%)$ & $31(75.6 \%)$ & 0.872 \\
\hline $\mathrm{BMI}, \mathrm{kg} / \mathrm{m}^{2}$ & $24.3 \pm 4.9$ & $23.2 \pm 3.6$ & 0.239 \\
\hline Smoking, $\mathrm{n}(\%)$ & $16(20.5 \%)$ & $11(26.8 \%)$ & 0.434 \\
\hline HTN, n(\%) & $61(78.2 \%)$ & $31(75.6 \%)$ & 0.748 \\
\hline $\mathrm{DM}, \mathrm{n}(\%)$ & $33(42.3 \%)$ & $15(36.6 \%)$ & 0.545 \\
\hline$A F, n(\%)$ & $39(50.0 \%)$ & $18(43.9 \%)$ & 0.527 \\
\hline Hyperlipidemia, n(\%) & $17(21.8 \%)$ & $12(29.3 \%)$ & 0.367 \\
\hline IHD, n(\%) & $36(46.2 \%)$ & $24(58.5 \%)$ & 0.199 \\
\hline HFrEF, n(\%) & $40(51.3 \%)$ & $18(43.9 \%)$ & 0.444 \\
\hline LVEF, \% & $40.7 \pm 14.9$ & $39.5 \pm 16.8$ & 0.720 \\
\hline \multicolumn{4}{|l|}{ Laboratory data } \\
\hline $\mathrm{HDL}-\mathrm{C}, \mathrm{mg} / \mathrm{dL}$ & $40.5 \pm 18.3$ & $49.1 \pm 19.6$ & 0.065 \\
\hline $\mathrm{LDL}-\mathrm{C}, \mathrm{mg} / \mathrm{dL}$ & $84.3 \pm 28.8$ & $96.5 \pm 32.0$ & 0.089 \\
\hline hs-CRP, mg/dL & $1.1 \pm 1.1$ & $1.4 \pm 2.0$ & 0.256 \\
\hline Troponin-I, ng/mL & $0.1 \pm 0.1$ & $0.2 \pm 0.3$ & 0.059 \\
\hline NT-pro-BNP, pg/ml & $3554 . \pm 3003.9$ & $4861.5 \pm 5453.0$ & 0.247 \\
\hline Creatinine $_{\text {admission }}, \mathrm{mg} / \mathrm{dL}$ & $1.8 \pm 0.9$ & $1.5 \pm 0.7$ & 0.092 \\
\hline Creatinine $_{\text {predischarge }}, \mathrm{mg} / \mathrm{dL}$ & $1.3 \pm 0.6$ & $1.8 \pm 0.8$ & 0.002 \\
\hline eGFR $\mathrm{admission}, \mathrm{mL} / \mathrm{min} / 1.73 \mathrm{~m}^{2}$ & $48.2 \pm 24.8$ & $55.4 \pm 27.6$ & 0.170 \\
\hline eGFR $_{\text {predischarge }}, \mathrm{mL} / \mathrm{min} / 1.73 \mathrm{~m}^{2}$ & $65.8 \pm 32.2$ & $46.8 \pm 25.6$ & 0.001 \\
\hline eGFR change, $\mathrm{mL} / \mathrm{min} / 1.73 \mathrm{~m}^{2}$ & $0.4 \pm 0.5$ & $-0.2 \pm 0.1$ & $<0.001$ \\
\hline
\end{tabular}

ACEl, angiotensin-converting enzyme inhibitor; $A F$, atrial fibrillation; $A R B$, angiotensin receptor blocker; $\mathrm{BMI}$, body mass index; DM, diabetes mellitus; eGFR, estimated glomerular filtration rate; HDL-C, highdensity lipoprotein-cholesterol; HFrEF, heart failure with reduced ejection fraction; $\mathrm{HTN}$, hypertension; hs-CRP, high sensitivity C-reactive protein; IHD, ischemic heart disease; LDL-C, low-density lipoproteincholesterol; LVEF, left ventricular ejection fraction; NT-pro-BNP, N-terminal pro-brain natriuretic peptide. 


\begin{tabular}{|c|c|c|c|}
\hline & No eGFR decline $(n=78)$ & eGFR decline $(n=41)$ & $P$-value \\
\hline \multicolumn{4}{|l|}{ Predischarge medication } \\
\hline Diuretics, n(\%) & $58(74.4 \%)$ & $37(90.2 \%)$ & 0.040 \\
\hline ACEI/ARB, n(\%) & $54(69.2 \%)$ & $31(75.6 \%)$ & 0.464 \\
\hline Beta-blockers, n(\%) & $36(46.2 \%)$ & $20(48.8 \%)$ & 0.785 \\
\hline Digitalis, n(\%) & $22(28.2 \%)$ & $5(12.2 \%)$ & 0.048 \\
\hline Vasodilators, n(\%) & $39(50.0 \%)$ & $24(58.5 \%)$ & 0.375 \\
\hline Follow-up duration (years) & $2.9 \pm 3.4$ & $2.0 \pm 2.5$ & 0.106 \\
\hline \multicolumn{4}{|c|}{$\begin{array}{l}\text { ACEI, angiotensin-converting enzyme inhibitor; AF, atrial fibrillation; ARB, angiotensin receptor blocker; } \\
\text { BMI, body mass index; DM, diabetes mellitus; eGFR, estimated glomerular filtration rate; HDL-C, high- } \\
\text { density lipoprotein-cholesterol; HFrEF, heart failure with reduced ejection fraction; HTN, hypertension; } \\
\text { hs-CRP, high sensitivity C-reactive protein; IHD, ischemic heart disease; LDL-C, low-density lipoprotein- } \\
\text { cholesterol; LVEF, left ventricular ejection fraction; NT-pro-BNP, N-terminal pro-brain natriuretic peptide. }\end{array}$} \\
\hline
\end{tabular}

\section{Renal function and outcomes in patients with ADHF}

During an average follow-up period of 2.6 \pm 3.2 years, 66 patients experienced 4P-MACE, including seven patients with CV death, four patients with nonfatal Ml, five patients with nonfatal stroke, and 50 patients

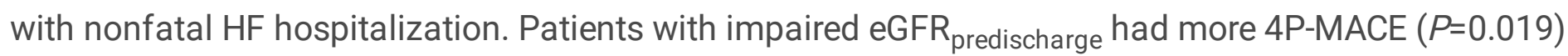
and HF hospitalization ( $P=0.042)$ than those with preserved eGFR predischarge (Table 5). Otherwise, the outcomes in patients with preserved eGFR admission $_{\text {and impaired eGFR }}$ admission were similar; the outcomes in patients with eGFR decline were also similar to those in patients without eGFR decline. 
Table 5

Outcomes according to renal function

\begin{tabular}{|c|c|c|c|}
\hline & Preserved eGFR admission $_{\text {. }}$ & Impaired eGFR $\mathrm{admission}$ & $P$-value \\
\hline CV death, n(\%) & $4(11.4 \%)$ & $3(3.6 \%)$ & 0.112 \\
\hline Nonfatal MI, n(\%) & $0(0.0 \%)$ & $4(4.8 \%)$ & 0.243 \\
\hline Nonfatal stroke, n(\%) & $0(0.0 \%)$ & $5(6.0 \%)$ & 0.169 \\
\hline Nonfatal HF, n(\%) & $12(34.3 \%)$ & $38(45.2 \%)$ & 0.270 \\
\hline \multirow[t]{2}{*}{ 4P-MACE, n(\%) } & $16(45.7 \%)$ & $50(59.5 \%)$ & 0.167 \\
\hline & Preserved eGFR $_{\text {predischarge }}$ & Impaired eGFR predischarge & P-value \\
\hline CV death, n(\%) & $4(7.8 \%)$ & $3(4.4 \%)$ & 0.343 \\
\hline Nonfatal MI, n(\%) & $1(2.0 \%)$ & $3(4.4 \%)$ & 0.424 \\
\hline Nonfatal stroke, n(\%) & $1(2.0 \%)$ & $4(5.9 \%)$ & 0.284 \\
\hline Nonfatal HF, n(\%) & $16(31.4 \%)$ & $34(50.0 \%)$ & 0.042 \\
\hline \multirow[t]{2}{*}{ 4P-MACE, n(\%) } & $22(43.1 \%)$ & $44(64.7 \%)$ & 0.019 \\
\hline & No eGFR decline & eGFR decline & P-value \\
\hline CV death, n(\%) & $5(6.4 \%)$ & $2(4.9 \%)$ & 0.544 \\
\hline Nonfatal MI, n(\%) & $2(2.6 \%)$ & $2(4.9 \%)$ & 0.428 \\
\hline Nonfatal stroke, n(\%) & $3(3.8 \%)$ & $2(4.9 \%)$ & 0.564 \\
\hline Nonfatal HF, n(\%) & 31 (39.7\%) & $19(46.3 \%)$ & 0.488 \\
\hline 4P-MACE, n(\%) & $41(52.6 \%)$ & $25(61.0 \%)$ & 0.248 \\
\hline
\end{tabular}

The Kaplan-Meier survival curves and log-rank test were used to identify the number of participants who did not develop 4P-MACE during the follow-up period. Although there was a trend of higher 4P-MACE in patients with impaired eGFR admission than in those with preserved eGFR admission, the difference was not significant $(P=0.125)$ (Figure 1). The incidence of $4 \mathrm{P}-\mathrm{MACE}$ was significantly higher in patients with impaired eGFR predischarge $_{\text {than in those with preserved eGFR predischarge }}(P=0.002)$ (Figure 2). The incidence of 4P-MACE was similar in patients with and without eGFR decline $(P=0.115)$ (Figure 3$)$.

Cox regression analysis revealed that in addition to age, impaired eGFR predischarge was significantly correlated with the development of 4P-MACE (HR, 2.003; 95\% Cl, 1.072-3.744; $P=0.029)$ (Table 6). Neither 


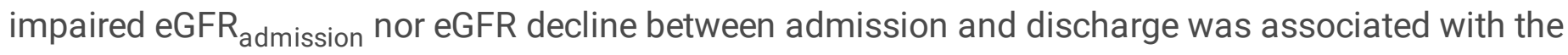
development of 4P-MACE. 
Table 6

Multivariate Cox proportional hazard analysis

\begin{tabular}{|c|c|c|c|}
\hline & HR & $(95 \% \mathrm{Cl})$ & $P$-value \\
\hline Age, years & 1.048 & $(1.023-1.074)$ & $<0.001$ \\
\hline Gender (male vs. female) & 0.858 & $(0.461-1.596)$ & 0.629 \\
\hline HTN (yes vs. no) & 0.747 & $(0.394-1.415)$ & 0.371 \\
\hline DM (yes vs. no) & 1.390 & $(0.793-2.436)$ & 0.250 \\
\hline IHD (yes vs. no) & 1.626 & $(0.963-2.744)$ & 0.069 \\
\hline ACEI/ARB (yes vs. no) & 0.757 & $(0.407-1.408)$ & 0.379 \\
\hline Beta-blocker (yes vs. no) & 1.504 & $(0.875-2.585)$ & 0.139 \\
\hline Digitalis (yes vs. no) & 1.179 & $(0.648-2.144)$ & 0.590 \\
\hline \multirow[t]{2}{*}{ Impaired GFR admission (yes vs. no) } & 1.405 & $(0.751-2.627)$ & 0.287 \\
\hline & HR & $(95 \% \mathrm{Cl})$ & P-value \\
\hline Age, years & 1.046 & $(1.020-1.072)$ & $<0.001$ \\
\hline Gender (male vs. female) & 0.870 & $(0.466-1.625)$ & 0.662 \\
\hline HTN (yes vs. no) & 0.879 & $(0.472-1.639)$ & 0.686 \\
\hline DM (yes vs. no) & 1.180 & $(0.664-2.098)$ & 0.572 \\
\hline IHD (yes vs. no) & 1.562 & $(0.933-2.613)$ & 0.090 \\
\hline ACEI/ARB (yes vs. no) & 0.751 & $(0.410-1.378)$ & 0.355 \\
\hline Beta-blocker (yes vs. no) & 1.490 & $(0.866-2.563)$ & 0.150 \\
\hline Digitalis (yes vs. no) & 1.492 & $(0.786-2.832)$ & 0.221 \\
\hline \multirow[t]{2}{*}{ Impaired eGFR predischarge (yes vs. no) } & 2.003 & $(1.072-3.744)$ & 0.029 \\
\hline & HR & $(95 \% \mathrm{Cl})$ & P-value \\
\hline Age, years & 1.047 & $(1.021-1.073)$ & $<0.001$ \\
\hline Gender (male vs. female) & 0.875 & $(0.469-1.634)$ & 0.676 \\
\hline HTN (yes vs. no) & 0.862 & $(0.456-1.631)$ & 0.649 \\
\hline DM (yes vs. no) & 1.447 & $(0.832-2.517)$ & 0.191 \\
\hline
\end{tabular}

ACEl, angiotensin-converting enzyme inhibitor; $\mathrm{ARB}$, angiotensin receptor blocker; $\mathrm{Cl}$, confidence interval; DM, diabetes mellitus; eGFR, estimated glomerular filtration rate; HR, hazard ratio; HTN, hypertension; IHD, ischemic heart disease. 


\begin{tabular}{|lccc|}
\hline & HR & (95\% Cl) & P-value \\
\hline IHD (yes vs. no) & 1.682 & $(1.007-2.808)$ & 0.047 \\
\hline ACEI/ARB (yes vs. no) & 0.740 & $(0.404-1.356)$ & 0.330 \\
\hline Beta-blocker (yes vs. no) & 1.437 & $(0.838-2.464)$ & 0.188 \\
\hline Digitalis (yes vs. no) & 1.193 & $(0.650-2.188)$ & 0.569 \\
\hline eGFR decline (yes vs. no) & 1.257 & $(0.727-2.173)$ & 0.414 \\
\hline $\begin{array}{l}\text { ACEl, angiotensin-converting enzyme inhibitor; ARB, angiotensin receptor blocker; Cl, confidence } \\
\text { interval; DM, diabetes mellitus; eGFR, estimated glomerular filtration rate; HR, hazard ratio; HTN, } \\
\text { hypertension; IHD, ischemic heart disease. }\end{array}$ & & & \\
\hline
\end{tabular}

\section{Discussion}

In this study, we found that impaired renal function before discharge was independently associated with poor clinical outcomes in Chinese patients with ADHF. However, WRF during hospitalization was not related to clinical outcomes in these patients.

\section{Renal function impairment and outcomes in patients with ADHF}

Renal function impairment, which is common in patients with ADHF, is associated with poor clinical outcomes in these patients. ${ }^{4,22}$ In the CRIC study, ${ }^{4}$ focusing on a large US CKD population, the rate ratio for HF re-hospitalization within 30 days was 2.6- and 1.9-fold higher in eGFR 30-44 and <30 $\mathrm{mL} / \mathrm{min} / 1.73 \mathrm{~m}^{2}$, respectively, compared with eGFR $\geq 45 \mathrm{~mL} / \mathrm{min} / 1.73 \mathrm{~m}^{2}$. Heywood et al. ${ }^{22}$ reported that renal dysfunction at admission was associated with higher in-hospital mortality in 118,465 patients hospitalized with ADHF. In the present study, we found that impaired renal function before discharge, defined as eGFR predischarge $<60 \mathrm{~mL} / \mathrm{min} / 1.73 \mathrm{~m}^{2}$, was associated with poor outcomes in patients with $A D H F$, which were mostly driven by nonfatal HF. Although patients with impaired renal function had more comorbidities than those with preserved renal function, the findings were still consistent after adjusting for baseline comorbidities, including HTN, DM, and IHD. Interestingly, cardiac biomarkers, including troponin-I and NT-pro-BNP, were similar in patients with preserved and impaired renal function. The findings suggest that renal function itself, rather than comorbidities or the severity of $\mathrm{HF}$, is related to clinical outcomes in patients with ADHF.

\section{Definitions of WRF}

WRF is commonly observed in patients hospitalized for ADHF, either on admission or during hospitalization. The definitions of WRF vary among different studies. Some were defined by increased levels of creatinine, ${ }^{7,9-12,14-16}$ some were defined by an increased percentage of creatinine; ${ }^{8}$ and some 
were defined by both. $5,6,13$ Recently, eGFR (calculated using the CKD-EPI formula) has been used to define WRF. ${ }^{17,18}$ In the present study, we used eGFR (calculated using the MDRD formula) to evaluate changes in renal function during hospitalization, which had better performance than creatinine and is commonly used in our clinical practice. ${ }^{21}$ Using this definition, WRF during hospitalization was noted in 41 (34.5\%) of 119 patients, which meant that nearly two-thirds of the patients could have preserved or improved renal function after decongestion therapy. Interestingly, WRF was not associated with comorbidities in these patients. However, it was related to more diuretic use and less digitalis use before discharge in these patients.

\section{Impacts of WRF on clinical outcomes in patients with ADHF}

The impact of WRF during hospitalization for ADHF has been examined in numerous studies. Some studies have reported that WRF is associated with worse long-term outcomes. Gottlieb et al. ${ }^{5}$ reported that any WRF predicted increased in-hospital mortality and prolonged hospital stays in patients hospitalized for HF. In the Vasodilation in the Management of Acute Congestive Heart Failure (VMAC) trial, ${ }^{7}$ WRF was associated with a higher rate of 6 -month mortality $(37.9 \%$ vs. $18.8 \%, P<0.001)$ and length of hospitalization ( $11.8 \pm 9.1$ days vs. $8.3 \pm 7.1$ days, $P<0.001)$ in patients with HF. In the Organized Program to Initiate Lifesaving Treatment in Hospitalized Patients With Heart Failure (OPTIMIZE-HF) registry, ${ }^{9,} 10$ WRF was associated with a higher rate of 30 -day readmission $(21.8 \%$ vs. $20.6 \%$; $P=0.01), 30$ day mortality $(10.0 \%$ vs. $7.2 \%, P<0.001)$, and 1 -year mortality (HR, $1.12 ; 95 \% \mathrm{Cl}, 1.04-1.20, P=0.003)$ after HF admission. A prospective study of ADHF showed that patients with WRF had a poorer outcome, defined as re-hospitalization and post-discharge death, compared with patients without WRF (HR, 1.12; $95 \% \mathrm{Cl}, 1.02-1.22 ; P=0.015) .{ }^{11}$ Berra et al. ${ }^{12}$ reported that WRF was strongly associated with a higher risk of death or readmission within 1 year after discharge in patients hospitalized for $\mathrm{HF}(\mathrm{HR}, 1.24 ; 95 \% \mathrm{Cl}$, 1.06-1.45, $P=0.0059$ ). Other related studies have also suggested that HF patients with WRF were likely to have a prolonged length of hospital stay, increased healthcare costs, increased in-hospital mortality, and higher rates of re-hospitalization and post-discharge death. ${ }^{6}$

In contrast, some studies have demonstrated that WRF is not necessarily associated with clinical outcomes in patients with HF. In a prospective multicenter study, ${ }^{14}$ patients with WRF had longer duration admissions, but a similar mortality and re-hospitalization rate to those without WRF. In the Diuretic Optimization Strategies Evaluation (DOSE) trial, ${ }^{15}$ when under high dose diuretic treatment, WRF was not associated with the composite endpoint of death, re-hospitalization, or emergency room visit within 60 days when compared with patients with stable renal function. In the PROTECT study (Placebo-controlled Randomized Study of the Selective A1 Adenosine Receptor Antagonist Rolofylline for Patients Hospitalized with Acute Decompensated Heart Failure and Volume Overload to Assess Treatment Effect on Congestion and Renal Function study), ${ }^{16}$ WRF was found to be associated with longer length of admission and a higher risk of death or readmission for $\mathrm{CV}$ or renal reason within 30 days, only in patients who at the time of creatinine measurement were significantly congested. Using data from the Ultrafiltration in Decompensated Heart Failure with Cardiorenal Syndrome and DOSE trials, ${ }^{17}$ an in- 
hospital decline in eGFR was not significantly associated with the composite outcome of death or rehospitalization within 60 days; however, a decline in eGFR may be associated with better outcomes when NT-proBNP declined. Using data from the Efficacy of Vasopressin Antagonism in Heart Failure Outcome Study with Tolvaptan (EVEREST) ${ }^{18}$ acute declines in kidney function were associated with an increased risk of mortality and CV outcomes only in those patients who had worsened markers of decongestion. In this study, we found that WRF itself was not associated with CV death, nonfatal MI, nonfatal stroke, nonfatal HF hospitalization, or the composite endpoint of 4P-MACE in patients admitted for ADHF. However, it remains unclear whether WRF results in impaired renal function before discharge, which is related to poor outcomes in these patients.

\section{Race and ethnicity}

Racial differences in HF outcomes have been reported in previous studies, ${ }^{25-27}$ which revealed that black and white patients had worse outcomes than other ethnicities. Data from the National Inpatient Sample (NIS) in the USA revealed that the age-standardized HF hospitalization rate was highest in Blacks, followed by Hispanics, Whites, and Asian/Pacific Islanders; the inpatient mortality was highest for Whites. ${ }^{25}$ Data from the ARIC Community Surveillance Study ${ }^{26}$ showed that white patients had a significantly higher mortality at 1 year compared with black patients, and worse renal function served as an independent predictor of mortality in white patients. In a retrospective study involving 53,640 hospitalized HF patients, ${ }^{27}$ every increase in creatinine of $0.5 \mathrm{mg} / \mathrm{dL}$ was associated with a $10 \%$ increased risk in adjusted mortality for Blacks, compared with 15\% increased risk in Whites.

The prevalence and incidence of CKD in Taiwan are relatively high compared to those in other countries, and it is associated with all-cause mortality in Taiwan. ${ }^{23,24}$ In this study, impaired renal function, either upon admission or before discharge, was commonly noted in our patients (84 [70.6\%] and 68 [57.1\%], respectively). We found that impaired renal function before discharge was associated with up to two times the risk of 4P-MACE (HR, 2.003; 95\% Cl, 1.072-3.744; $P=0.029)$ in Chinese patients with ADHF. This finding is compatible with the risks reported in the CRIC study, ${ }^{4}$ which was mainly focused on nonHispanic Whites and non-Hispanic Blacks. This suggests that the impact of renal dysfunction in Chinese patients is as important as that in white and black patients. Since the relationships between ADHF and renal dysfunction have rarely been reported in the Chinese population, our study provides important information about risk stratification in patients with ADHF.

\section{Limitations}

This study has some limitations. First, this was a single-center study with a small study population. However, this is the first study to comprehensively investigate the association between renal function during hospitalization and clinical outcomes in Chinese patients with ADHF. Further studies with larger sample sizes are required. Second, we did not have information regarding baseline renal function before admission. Since most of the patients were newly diagnosed with HF and were experiencing their first hospitalization, they did not undergo any tests before admission. Furthermore, the study was designed to investigate renal function during hospitalization and clinical outcomes in patients with ADHF; only renal 
function tests during hospitalization were collected. The findings are more applicable to patients without previous hospital visits, experiencing their first HF hospitalization. Third, serum biomarkers, troponin-I, NTpro-BNP, and hs-CRP, were only measured once in this study. Therefore, we did not have information regarding the change in NT-pro-BNP, which was used as a marker of decongestion in previous studies. ${ }^{18}$ Further studies are needed to clarify the impact of biomarkers and their changes on clinical outcomes in this population.

\section{Conclusions}

Impaired renal function before discharge is a significant risk factor for poor clinical outcomes in Chinese patients with ADHF. However, WRF was not associated with clinical outcomes in these patients. Our study not only highlights the importance of renal function before discharge as a biomarker for risk stratification but also supports the safety of decongestion therapy for renal function in Chinese patients with ADHF.

\section{Declarations}

Acknowledgements:This work was supported by research grant V109C-076 from Taipei Veterans General Hospital, Taipei, Taiwan, R.O.C., and research grant MOST 108-2314-B-075-062-MY3 from the Ministry of Science and Technology, Taiwan, R.O.C. The funders played no role in data collection or preparation of the manuscript.

Author contributions: HWL contributed to conception and design, analysis and interpretation of data, and drafted the manuscript. CYY, HBL, PHH, TCW, SJL, and JWC contributed to data acquisition and drafted the manuscript. $\mathrm{CCH}$ contributed to conception, data acquisition, analysis and interpretation of data, drafted and critically revised the manuscript. All authors gave final approval and agreed to be accountable for all aspects of work ensuring integrity and accuracy.

Competing interests:The authors declare no competing interests.

Data availability: The datasets generated during and/or analysed during the current study are available from the corresponding author on reasonable request.

\section{References}

1. Schefold, J. C., Filippatos, G., Hasenfuss, G., Anker, S. D. \& von Haehling, S. Heart failure and kidney dysfunction: epidemiology, mechanisms and management. Nat. Rev. Nephrol, 12, 610-623 (2016).

2. Gudsoorkar, P. S. \& Thakar, C. V. Acute kidney injury, heart failure, and health outcomes. Cardiol. Clin, 37, 297-305 (2019).

3. Löfman, I., Szummer, K., Dahlström, U., Jernberg, T. \& Lund, L. H. Associations with and prognostic impact of chronic kidney disease in heart failure with preserved, mid-range, and reduced ejection fraction. Eur. J. Heart Fail, 19, 1606-1614 (2017). 
4. Bansal, N. et al. Burden and outcomes of heart failure hospitalizations in adults with chronic kidney disease. J. Am. Coll. Cardiol, 73, 2691-2700 (2019).

5. Gottlieb, S. S. et al. The prognostic importance of different definitions of worsening renal function in congestive heart failure. J. Card. Fail, 8, 136-141 (2002).

6. Smith, G. L. et al. Worsening renal function: what is a clinically meaningful change in creatinine during hospitalization with heart failure? J. Card. Fail, 9, 13-25 (2003).

7. Akhter, M. W. et al. Effect of elevated admission serum creatinine and its worsening on outcome in hospitalized patients with decompensated heart failure. Am. J. Cardiol, 94, 957-960 (2004).

8. Metra, M. et al. Worsening renal function in patients hospitalised for acute heart failure: clinical implications and prognostic significance. Eur. J. Heart Fail, 10, 188-195 (2008).

9. Patel, U. D. et al. Associations between worsening renal function and 30-day outcomes among Medicare beneficiaries hospitalized with heart failure. Am. Heart J. 160: 132-138.e1 (2010)

10. Kociol, R. D. et al. Long-term outcomes of medicare beneficiaries with worsening renal function during hospitalization for heart failure. Am. J. Cardiol, 105, 1786-1793 (2010).

11. Lanfear, D. E. et al. Relation of worsened renal function during hospitalization for heart failure to long-term outcomes and rehospitalization. Am. J. Cardiol, 107, 74-78 (2011).

12. Berra, G. et al. Outcome in acute heart failure: prognostic value of acute kidney injury and worsening renal function. J. Card. Fail, 21, 382-390 (2015).

13. Aronson, D. \& Burger, A. J. The relationship between transient and persistent worsening renal function and mortality in patients with acute decompensated heart failure. J. Card. Fail, 16, 541-547 (2010).

14. Cowie, M. R., Komajda, M., Murray-Thomas, T., Underwood, J. \& Ticho, B. POSH Investigators. Prevalence and impact of worsening renal function in patients hospitalized with decompensated heart failure: results of the prospective outcomes study in heart failure (POSH). Eur. Heart J, 27, 1216-1222 (2006).

15. Brisco, M. A. et al. Relevance of changes in serum creatinine during a heart failure trial of decongestive strategies: insights from the DOSE trial. J. Card. Fail, 22, 753-760 (2016).

16. Metra, M. et al. Prognostic significance of creatinine increases during an acute heart failure admission in patients with and without residual congestion: a post hoc analysis of the PROTECT data. Circ. Heart Fail, 11, e004644 (2018).

17. McCallum, W., Tighiouart, H., Kiernan, M. S., Huggins, G. S. \& Sarnak, M. J. Relation of kidney function decline and NT-proBNP with risk of mortality and readmission in acute decompensated heart failure. Am. J. Med. 133: 115-122.e2 (2020)

18. McCallum, W. et al. Acute kidney function declines in the context of decongestion in acute decompensated heart failure. JACC. Heart Fail, 8, 537-547 (2020).

19. Ponikowski, P. et al. 2016 ESC Guidelines for the diagnosis and treatment of acute and chronic heart failure: The Task Force for the diagnosis and treatment of acute and chronic heart failure of the 
European Society of Cardiology (ESC) Developed with the special contribution of the Heart Failure Association (HFA) of the ESC. Eur. Heart J, 37, 2129-2200 (2016).

20. Yancy, C. W. et al. 2017 ACC/AHA/HFSA focused update of the 2013 ACCF/AHA guideline for the management of heart failure: a report of the American College of Cardiology/American Heart Association Task Force on Clinical Practice Guidelines and the Heart Failure Society of America., 136, e137-e161 (2017).

21. Levey, A. S. et al. A more accurate method to estimate glomerular filtration rate from serum creatinine: a new prediction equation. Modification of Diet in Renal Disease Study Group. Ann. Intern. Med, 130, 461-470 (1999).

22. Heywood, J. T. et al. High prevalence of renal dysfunction and its impact on outcome in 118,465 patients hospitalized with acute decompensated heart failure: a report from the ADHERE database. J. Card. Fail, 13, 422-430 (2007).

23. Kuo, H. W., Tsai, S. S., Tiao, M. M. \& Yang, C. Y. Epidemiological features of CKD in Taiwan. Am. J. Kidney Dis, 49, 46-55 (2007).

24. Wen, C. P. et al. All-cause mortality attributable to chronic kidney disease: a prospective cohort study based on 462293 adults in Taiwan., 371, 2173-2182 (2008).

25. Ziaeian, B. et al. National differences in trends for heart failure hospitalizations by sex and race/ethnicity. Circ. Cardiovasc. Qual. Outcomes, 10, e003552 (2017).

26. Sharma, K. et al. Predictors of mortality by sex and race in heart failure with preserved ejection fraction: ARIC community surveillance study. J. Am. Heart Assoc, 9, e014669 (2020).

27. Smith, G. L. et al. Race and renal impairment in heart failure: mortality in blacks versus whites., 111, 1270-1277 (2005).

\section{Figures}




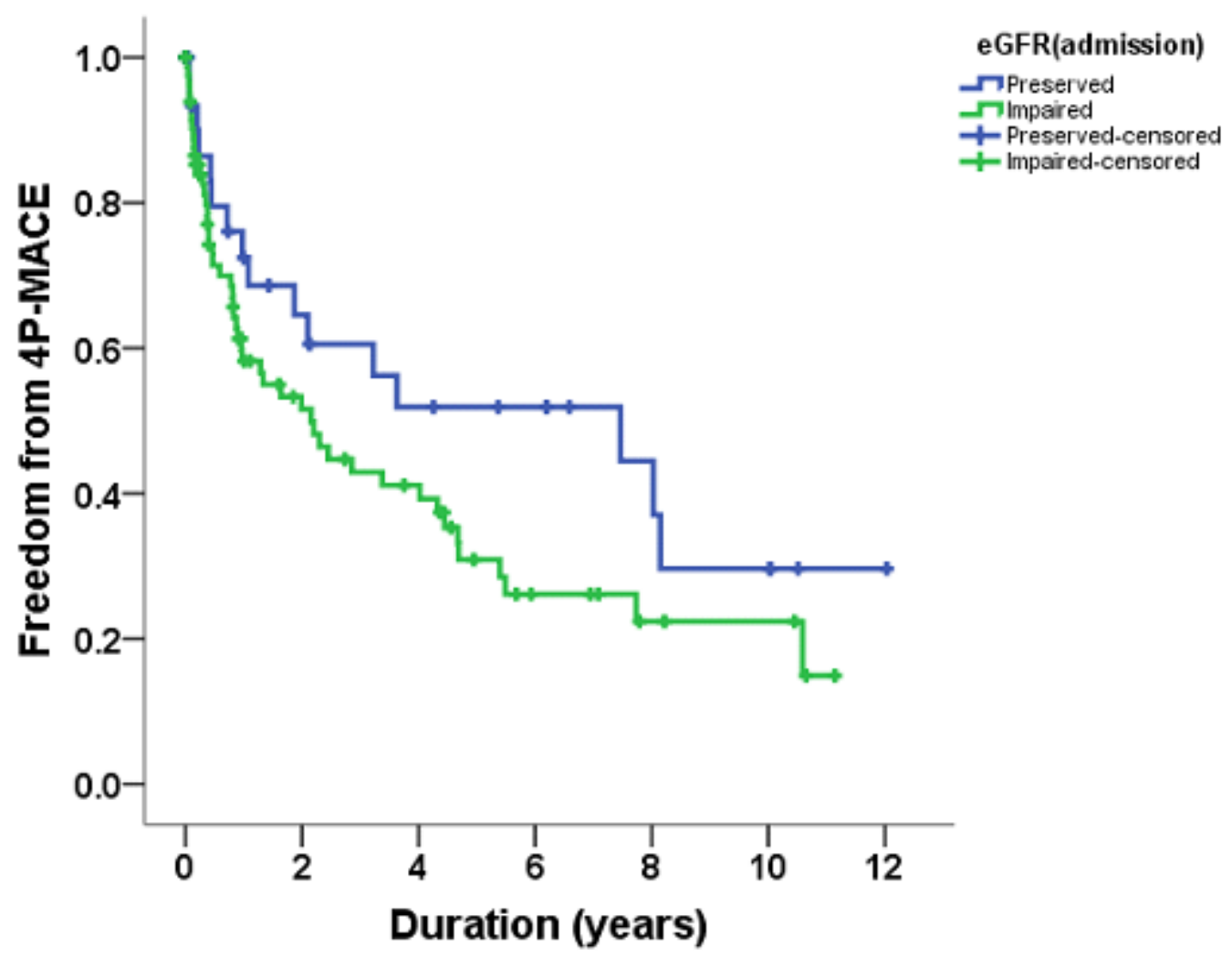

Number at risk: eGFR (adm/sion)

$\begin{array}{llllllll}\text { Preserved } & 35 & 16 & 12 & 10 & 6 & 4 & 1 \\ \text { Impaired } & 84 & 30 & 22 & 9 & 5 & 4 & 0\end{array}$

\section{Figure 1}

Kaplan-Meier survival curves showing the absence of 4P-MACE according to eGFRadmission in patients with ADHF. All participants were divided into two groups according to eGFRadmission. The blue line represents the patient group with preserved eGFRadmission (eGFRadmission $\geq 60 \mathrm{~mL} / \mathrm{min} / 1.73 \mathrm{~m} 2$ ). The green line represents the patient group with impaired eGFRadmission (eGFRadmission $<60$ $\mathrm{mL} / \mathrm{min} / 1.73 \mathrm{~m} 2)$. Differences were compared using the log-rank test $(P=0.125)$. 


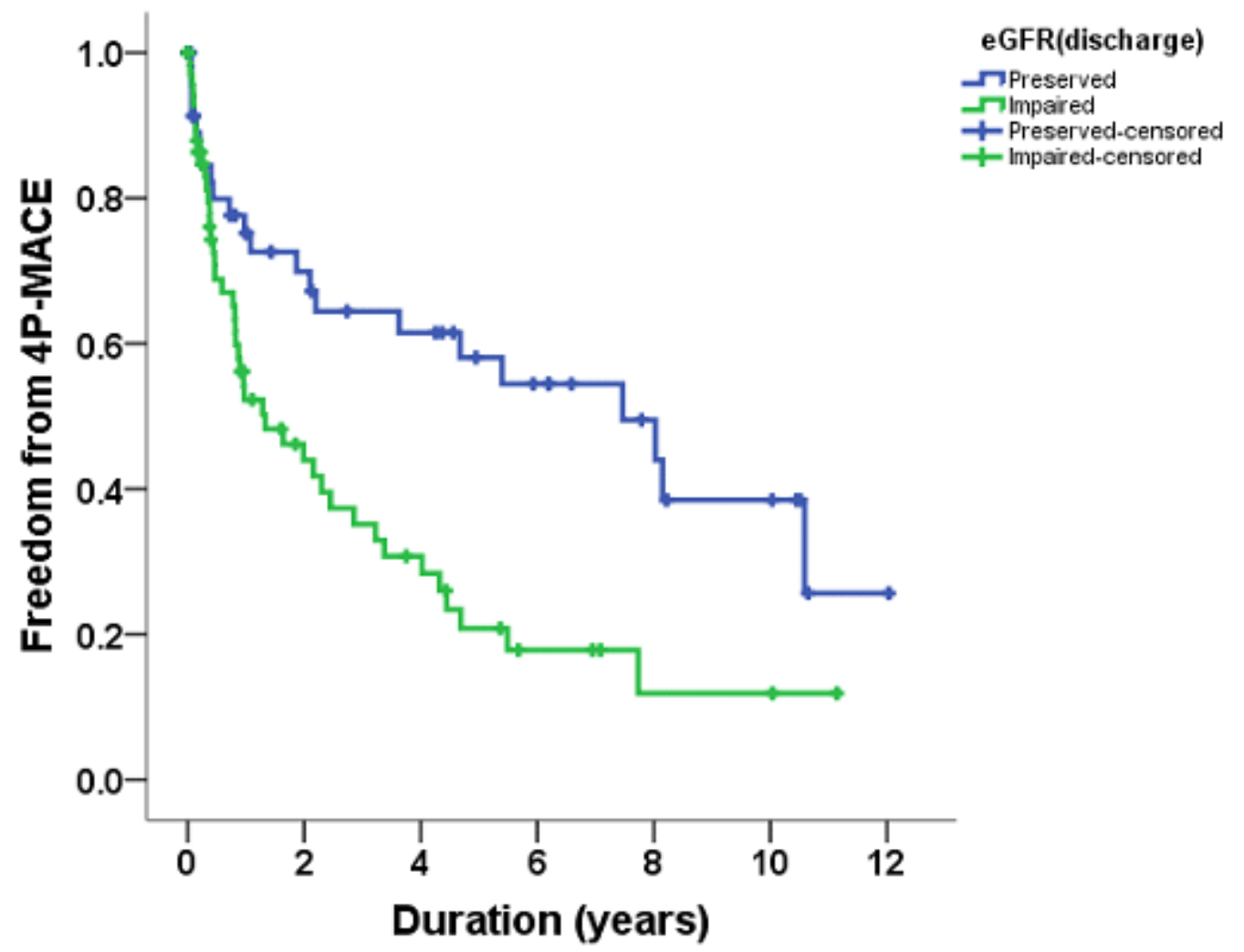

Number at risk: eGFR (dlscharge)

$\begin{array}{llllllll}\text { Preserved } & 51 & 26 & 21 & 14 & 9 & 6 & 1 \\ \text { Impaired } & 68 & 20 & 13 & 5 & 2 & 2 & 0\end{array}$

\section{Figure 2}

Kaplan-Meier survival curves showing the absence of 4P-MACE according to eGFRdischarge in patients with ADHF. All participants were divided into two groups according to eGFRpredischarge. The blue line represents the patient group with preserved eGFRdischarge (eGFRpredischarge $\geq 60 \mathrm{~mL} / \mathrm{min} / 1.73 \mathrm{~m} 2$ ). The green line represents the patient group with impaired eGFRpredischarge (eGFRpredischarge $<60$ $\mathrm{mL} / \mathrm{min} / 1.73 \mathrm{~m} 2)$. Differences were compared using the log-rank test $(P=0.002)$. 


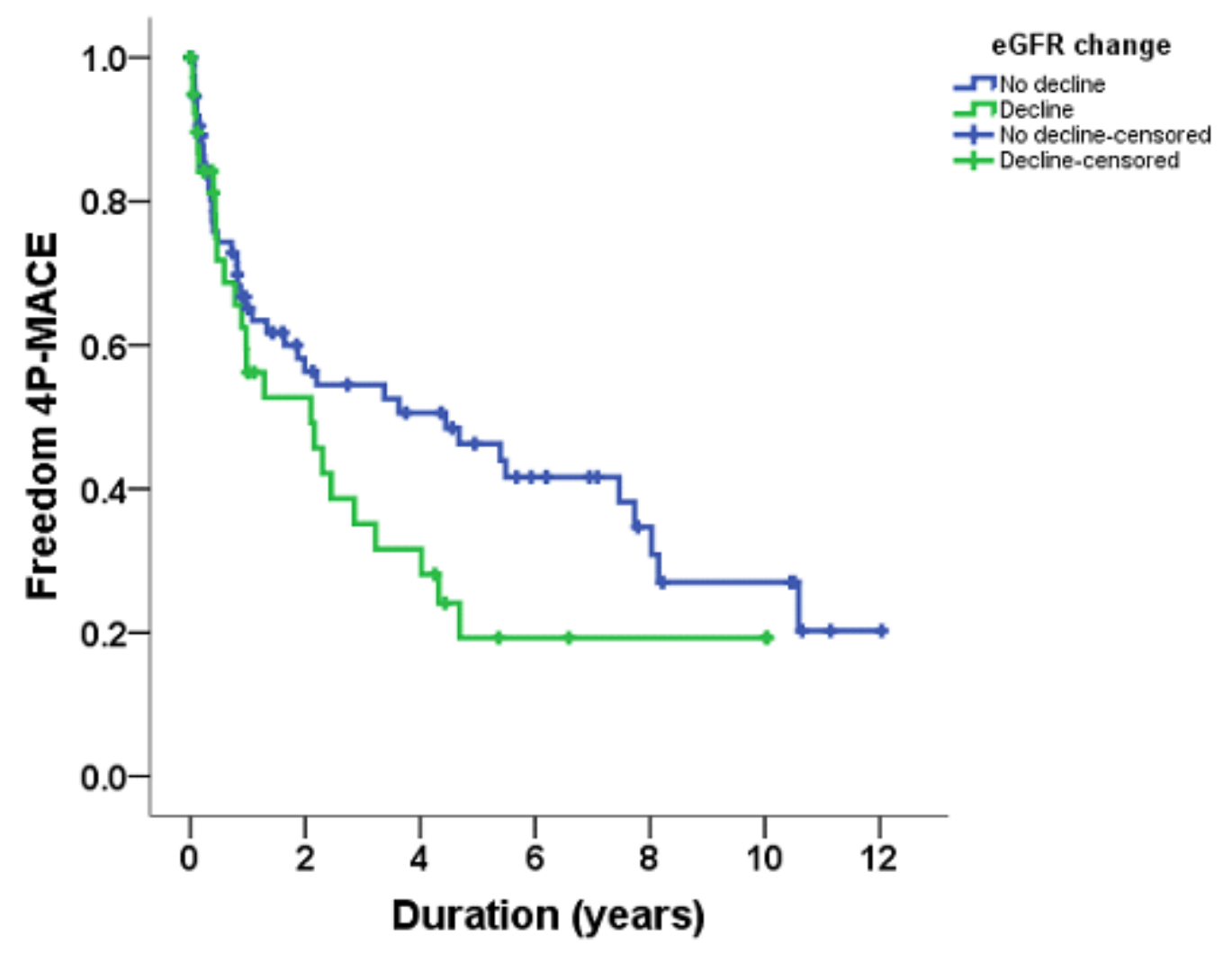

Number at risk: eGFR change

$\begin{array}{llllllll}\text { No decline } & 78 & 31 & 25 & 16 & 9 & 6 & 1 \\ \text { Dedine } & 41 & 15 & 9 & 3 & 2 & 2 & 0\end{array}$

Figure 3

Kaplan-Meier survival curves showing the absence of 4P-MACE according to eGFR change between admission and pre-discharge in patients with ADHF. All participants were divided into two groups according to eGFR change. The blue line represents the patient group without eGFR decline. The green line represents the patient group with eGFR decline. Differences were compared using the log-rank test $(P=0.115)$.

\section{Supplementary Files}

This is a list of supplementary files associated with this preprint. Click to download.

- 20211120CKDandHFSupplementaryTable1.docx

- 20211120CKDandHFSupplementaryTable2.docx

- 20211120CKDandHFSupplementaryTable3.docx 